\title{
A Methodology for Optimizing the Process of Machining a Workpiece Using Multi-Objective Particle Swarm Optimization
}

\author{
Osslan Osiris Vergara Villegas, Carlos Felipe Ramírez Espinoza, Vianey Guadalupe \\ Cruz Sánchez, Manuel Nandayapa, Jorge Luis García-Alcaraz
}

Universidad Autónoma de Ciudad Juárez, Instituto de Ingeniería y Tecnología, Ciudad Juarez, Chihuahua, México

\{overgara, caramire, vianey.cruz, mnandaya, jorge.garcia\}@uacj.mx

\begin{abstract}
In this paper a methodology based on multi-objective particle swarm optimization algorithm, for identifying the optimal parameters for machining a workpiece with a milling is presented. The time for machining, the material removal rate, and the feed rate were identified as the objectives to optimize. In addition, the proposal considered 4 constraints related to cutting tools, rotating speed of main spindle, cutting depth per passing, and speed interval for advance. Once the objective functions and constraints were determined, the test workpiece was designed by an unexperienced machinist by means of CATIA software, and then exported to Mastercam X in order to generate the G \& M codes. The material selected for machining was delrin. In the experimentation stage, the methodology proposed was executed 50 times, and the parameters from the 2 best solutions were used to design 2 new workpieces. From the results obtained it was observed that the methodology proposed can support unexperienced operators in optimizing the parameters for machining. The machining time was reduced in $30 \%$, material removal rate was increased about $55 \%$, and an increment of $14 \%$ was obtained for the feed rate.
\end{abstract}

\section{Introduction}

Manufacturing is the process of converting, by hand or by machine, raw materials, components, or parts into finished goods that meet a customer's specifications. It implies, the application of physical and chemical processes to alter the geometry, properties, and appearance of a given starting material to make parts or products [1]. Manufacturing operations can be broadly classified into two different groups called processing, and assembly. The former, transforms a work material to the final desired product. The later, joins two or more components to create a new entity called an assembly or subassembly.

An important task inside processing operations is the material removal or well known as machining. Machining is the manufacturing process in which a cutting tool is used to remove the material excess from a workpiece, in such a way that the reminder obtains a desired shape. The machining process can be divided into two groups which 
are: a) cutting process with traditional machining in which turning, milling, boring, and grinding are included, and b) cutting process with modern machining in which electrical discharge machining (EDM), and abrasive waterjet (AWJ) are included [2].

Typically, when a workpiece will be machined, a process planer selects the machining parameters based on his experience, and from the available handbooks. In the literature, a number of recommended machining parameters can be found, however, most of times those parameters do not suits exactly for a particular machine tool, material, and other combinations [3]. The poor selection of machining parameters leads to several drawbacks such as long machining times, and large waste of materials, therefore, it is imperative to enhance the parameters involved in workpiece machining. One of the main goals in machining is to produce high quality products with less cost and time constraints, and this can be due by optimizing the selection of process parameters such as cutting speed, depth of cut, feed rate, to mention a few. In several fields such as computer science, artificial intelligence, operation research, and manufacturing, optimization is the process of trying to find the best possible solution to a problem [4].

Several traditional and non-traditional methods can be used for determining the optimal machining parameters. Traditional techniques include dynamic programming, geometric programming, and deterministic techniques. However, these techniques either tend to result in local minima or take a long time to converge to an acceptable result [5]. On the other hand, non-traditional techniques include genetic algorithm (GA), simulated annealing (SA) [6], particle swarm optimization (PSO) [7], ant colony optimization (ACO) [8], and artificial bee colony (ABC) [9].

In the literature a number of single objective approaches have been presented for optimization of machining parameters such as material removal rate (MRR), surface roughness, cutting force, tool life, power consumption, to mention a few. However, several single objective problems must be applied to optimize the number of different criterions involved in machining processes. On the other hand, multi-objective approaches can be used to optimize several criterions at a time. In multi-objective optimization problems, the objectives to optimize are normally in conflict with respect to each other, therefore, there is no single solution to these problems. Hence, good trade-off solutions that represent the best possible compromises among objectives is frequently obtained. The trade-off solutions are called Pareto optimal set [10].

A reduced number of works, can be found in the literature regard multi-objective optimization of machining parameters. The paper of [11], considers the environmental dimension for optimizing milling cutting operations; three objectives, such as surface roughness, MRR, and cutting energy, were simultaneously optimized. A multiobjective optimization of lathe machining parameters for energy saving was shown in the work of [12], where three objectives including energy, cost, and quality were considered, which were affected by 3 constraints, namely cutting depth, feed rate, and cutting speed. A software prototype for solving multi-objective machining optimization problems was developed in [13]; the core was an algorithm based on exhaustive iterative search which guarantees the optimality of a determined solution in a given discrete search space, a wire electric discharge machining (WEDM), a micro WEDM, and a laser machining were considered for tests. The problem of computing the Pareto front was addressed in the paper of [14], by enumerating and characterizing 128 scenarios in sustainable machining operation involving 7 objectives including energy, 
cost, time, power, cutting force, tool life and surface finish; several recommendations were offered to create a generic optimization framework. Finally, a method for complex optimization of cutting parameters with the objectives of energy efficiency and milling processing time, which integrates Taguchi method, response surface method (RSM), and multi-objective particle swarm optimization algorithm (MOPSO) was presented in [7].

Motivated from the revision above, and by observing that multi-objective methods have gained great attention, in this paper a method for multi-objective optimization of objective functions related to machining time, MRR, and feed rate for a milling machine is presented. The proposal considered 4 constraints related to rotating speed of main spindle, cutting depth per passing, speed interval for the advance, and cutting tools. Therefore, the primary focus of this paper is to suggest procedures to determine the optimal machining parameters for single-pass milling of a workpiece.

\section{Proposed Methodology}

The methodology proposed comprises two main stages; the first one, related to machining the workpiece, and the second one, related to MOPSO.

\subsection{Machining the Workpiece}

The first step in the methodology proposed, consists of machining a test workpiece with a specific geometry, by means of a computerized numerical control (CNC) machine. The milling selected was the vertical VIWA VF3KM400, which have a 5 horsepower (HP) triphasic motor, table dimensions of $1270 \times 254 \mathrm{~mm}$, spindle career of $120 \mathrm{~mm}$, and variable speed range from 120-4200 revolutions per minute (rpm). A graphical example of the milling used is shown in Figure 1.

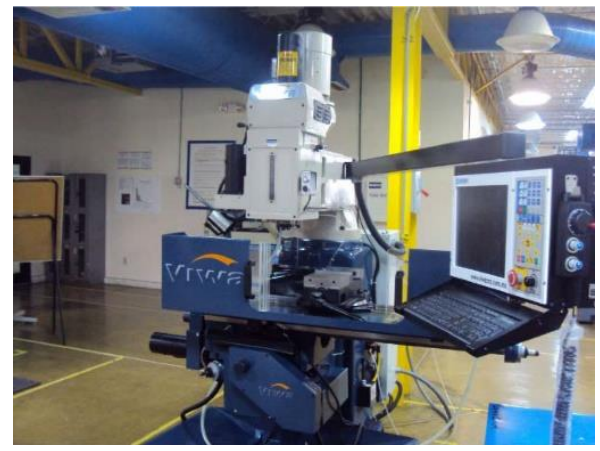

Fig. 1. The milling machine used for piece machining.

The material selected to build the workpiece was Polyoxymethylene (POM), better known as delrin, which was prepared by means of cutting, and carious operations. The cutting process started from a solid delrin block with a size of $65 \mathrm{~mm} \times 65 \mathrm{~mm} \times$ $50.8 \mathrm{~mm}$. The workpiece was made up of a cylinder placed at the center, with a diameter and a height of $30 \mathrm{~mm}$. The cylinder contains a hole of $12.5 \mathrm{~mm} \times 12.5 \mathrm{~mm}$ with a depth 
of $15 \mathrm{~mm}$. In addition, 4 holes were created in the corners of the piece, with a diameter and depth of $10 \mathrm{~mm}$.

The workpiece was designed by an unexperienced user by means of the computer aided three-dimensional interactive application (CATIA) software. The piece designed in CATIA was exported to Mastercam X in order to define the methodology, the tools, and the necessary parameters to perform the machining in a virtual way. The goal of this was to verify the existence of possible errors before the real workpiece machining. The workpiece designed in CATIA, and its correspondent representation in Mastercam $\mathrm{X}$ are shown in Figure 2.

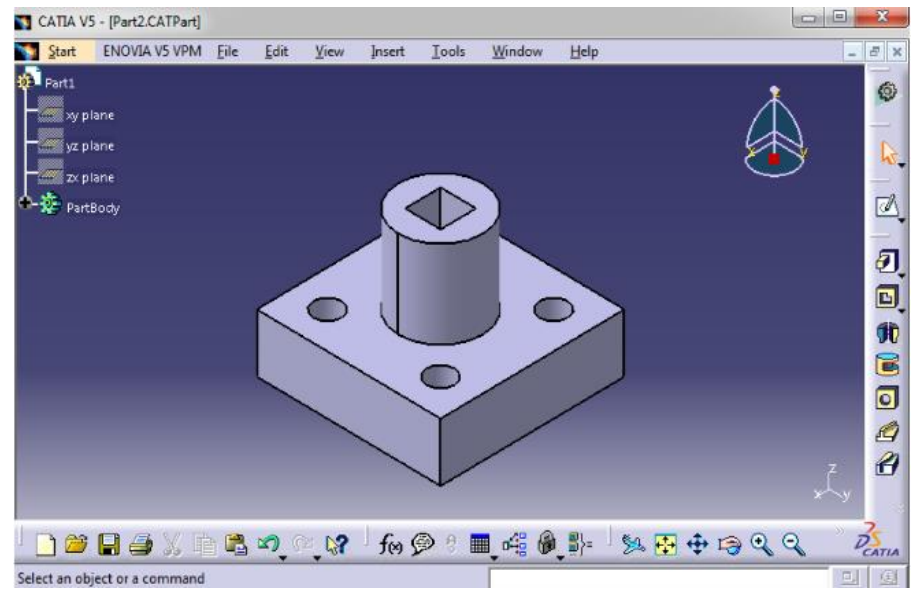

a)

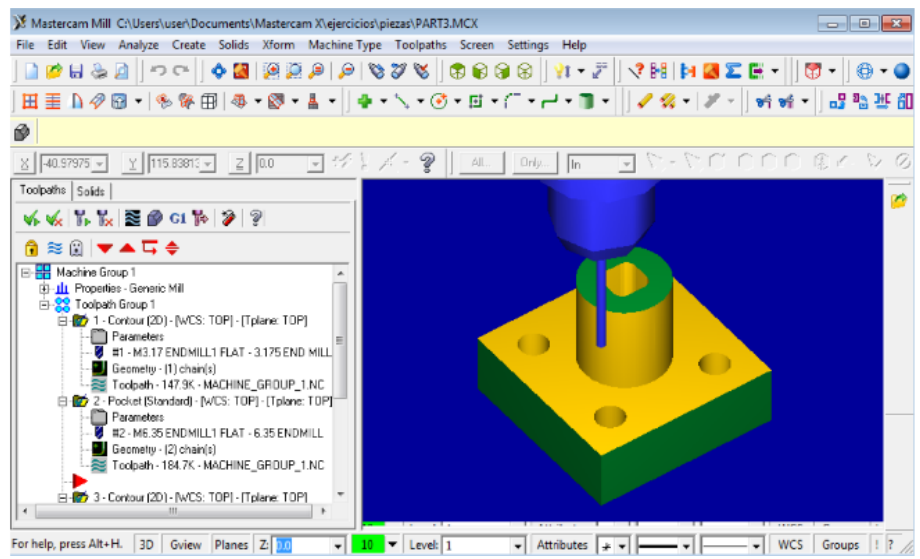

b)

Fig. 2. The workpiece designed in: a) CATIA, and b) Mastercam X.

Once that the correct design of the piece was virtually verified, the $\mathrm{G}$ and $\mathrm{M}$ codes were created, and used to control and drives the milling. The parameters used for the initial workpiece machining were set to: depth from 1.5 to $3 \mathrm{~mm}$, cutters with 2 and 4 teeth, with a size of $1 / 8$ and $1 / 4$ respectively, and 0.073 millimeters per tooth $m m P T$, 
and rotating speed of main spindle of $3000 \mathrm{rpm}$. The final workpiece designed is shown in Figure 3.

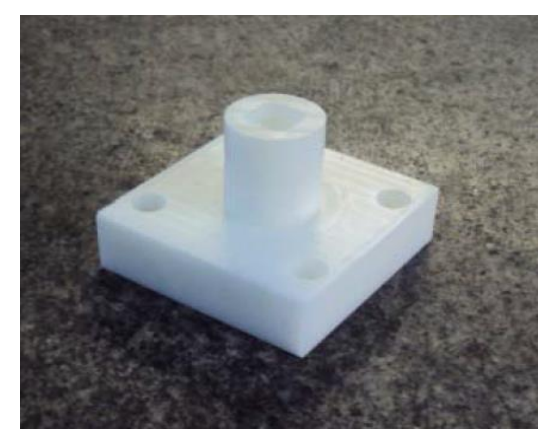

Fig. 3. The final workpiece designed.

\subsection{Multi-Objective Particle Swarm Optimization}

The metaheuristic used to solve the multi-objective optimization problem of this paper is known as PSO, that simulates the movements of a flock of birds which aim to find food. The main advantages of PSO includes better exploration and exploitation provided by local and global search capabilities of the algorithm.

According to [15], PSO starts with the random initialization of a population (swarm) of individuals (particles) in the $n$-dimensional search space ( $n$ is the dimension of problem). The particles fly over search space with adjusted velocities. Each particle keeps two values in its memory; its own best experience, and the best experience of the whole swarm.

2.2.1 Definition of the Objective Functions. Three different objective functions were defined in order to optimize the time for machining a piece (TM), the $M R R$, and the feed rate $(f)$. It is important to mention that the three functions were merged in a sum function.

The function related to $T M$ expressed in $\min$ is shown in equation 1:

$$
T M=\sum_{n=1}^{T} t_{n} .
$$

The function of $M R R$ expressed in $\mathrm{cm}^{3} / \mathrm{min}$ is shown in equation 2:

$$
M R=n \times m m P T \times W \times A \times Z,
$$

where $n$ is the rotating speed of spindle measured in rpm, $m m P T$ are the millimeters per tooth of the cutting tool, $W$ is the radial width of cut, $A$ is the axial depth of cut, and $Z$ is the number of cutting tool teeth.

The third objective function corresponds to $f$, and it is expressed in $\mathrm{mm} / \mathrm{min}$ as shown in equation 3:

$$
f=n \times m m P T \times Z .
$$


2.2.2 Constraints. The constraints defined for the proposed model were the following:

1. The rotating speed of main spindle must rank in an interval of 2500-4000 rpm, because it represents the middle to high rank of the milling machine.

2. The cutting depth per passing was defined in $1-4 \mathrm{~mm}$.

3. The speed interval for the advance was defined as 20-40 IPM.

4. Only high speed steel (HSS) cutting tools of 2 and 4 teeth were used, and the search space was limited to $1 / 16-5 / 16$.

2.2.3 PSO Parameters. The parameters employed for the PSO algorithm were:

- The initial swarm was set to 10 particles.

- The maximum number of iterations was set to 50 .

- The maximum and minimal speed $\left[-\mathrm{V}_{\max }, \mathrm{V}_{\max }\right]$ interval was set to $[-5,5]$.

- The inertia factor decrement was realized with a maximum value of 1.4 , and a minimal value of 0.4 .

- $\quad$ The learning factors values of $c_{1}$ and $c_{2}$ were set to 1.49618 .

\section{Experimental Results}

The initial parameters for machining the workpiece were inserted as inputs to PSO, and then the proposed algorithm was executed. Once that the stop criterion was met, an array containing the better solutions is delivered as an output.

For the experimentation, the two best solutions from the set obtained were selected, after that, by using the optimized parameters two new pieces were machined. The parameters obtained for the best solution (MOPSO1) were cutting depth of $3.5 \mathrm{~mm}$, cutting tool with a size of $1 / 4,4$ teeth, and $0.0656 \mathrm{mmPT}$, and rotating speed of 3388 rpm. For the second better solution (MOPSO2), the parameters obtained were cutting depth of $4 \mathrm{~mm}$, cutting tool with a size of $1 / 4,4$ teeth, and $0.0810 \mathrm{mmPT}$, and rotating speed of $3266 \mathrm{rpm}$. A summary of the values obtained from experimentation is shown in Table 1. In addition, the percentage improvements are shown in Table 2.

Table 1. Results obtained from experiments

\begin{tabular}{lccc}
\hline \multicolumn{1}{c}{ Piece } & $\boldsymbol{T M}$ & $\boldsymbol{M R R}$ & $\boldsymbol{f}$ \\
\hline Original & $18.03 \mathrm{~min}$ & $12.554 \mathrm{~mm} / \mathrm{min}$ & $779.44 \mathrm{~cm}^{3} / \mathrm{min}$ \\
MOPSO1 & $12.23 \mathrm{~min}$ & $19.758 \mathrm{~mm} / \mathrm{min}$ & $889.01 \mathrm{~cm}^{3} / \mathrm{min}$ \\
MOPSO2 & $12.47 \mathrm{~min}$ & $18.709 \mathrm{~mm} / \mathrm{min}$ & $736.80 \mathrm{~cm}^{3} / \mathrm{min}$ \\
\hline
\end{tabular}

Table 2. Improvement percentages from experiments

\begin{tabular}{cccc}
\hline Piece & $\boldsymbol{T M}$ & $\boldsymbol{M R R}$ & $\boldsymbol{f}$ \\
\hline Original & - & - & - \\
MOPSO1 & $32.06 \%$ & $57.38 \%$ & $14.06 \%$ \\
MOPSO2 & $30.84 \%$ & $49.03 \%$ & $-5.47 \%$ \\
\hline
\end{tabular}




\subsection{Discussion}

It should be noted from Tables 2 and 3 that the methodology proposed for machining a workpiece with optimized parameters offers competitive results. The TM was reduced in both solutions in 6 min (from 18 to 12), which represent a competitive percentage reduction of about $30 \%$. The time reduction is mainly due to the increase of MRR, the faster the better for most machinist, therefore, the industry could deliver mores pieces by turn, and generate more economic gains.

The MRR was enhanced in $6 \mathrm{~mm} / \mathrm{min}$, which represent an approximate gain of $56 \%$. From the experiments it was observed that even with the increase of MRR the cutting tool life is not affected (due to the material used), no chipping was presented, and a good surface finish is obtained. In addition, it is important to note that for the case of MOPSO1, $f$ was enhanced from $779.44 \mathrm{~cm}^{3} / \mathrm{min}$ to $889.01 \mathrm{~cm}^{3} / \mathrm{min}$, which represent a gain of $14.06 \%$. However, as can be observed for the case of MOPSO2 no $f$ improvement was obtained, unlike a decrement of $11 \mathrm{~cm}^{3} / \mathrm{min}$ was obtained, even with this the other parameters influencing for good machining. In comparison with the literature works discussed in Section 1, the geometry of the workpiece machined in this paper is not trivial, therefore, the selection of the parameter by an unexperienced and even an experience machinist is complex. After the machining of the two workpieces with the parameters obtained with the methodology proposed, it was observed that its quality was good in terms of geometry, and surface finish.

\section{Conclusions}

In this paper a methodology based on multi-objective particle swarm optimization algorithm, for identifying the optimal parameters for machining a workpiece with a milling was presented. The results obtained from experiment proved that the optimized machining parameters of PSO could yield to improve the machining time, the MRR, and the feed rate for a particular geometry of a workpiece, and obtaining a good surface finish. The optimization results obtained in this paper confirm that the proposed optimization method is a very useful tool for multi-objective optimization of machining parameters. In addition, the proposed methodology can solve the trade-offs well when objectives were generally conflicting to each other, and constraints must be fulfilled.

Future work will be directed towards on extending the current approach to include more different and complex workpiece geometries. Also, it will be important extend the proposal to a wider of optimization of machining parameters. Regards to the material, it will be interesting perform tests with other material such as aluminum, cast iron, graphite stainless steel, among others. Finally, the model could be extended with objective functions related to cutting tool life, and surface roughness.

\section{References}

1. Groover, M.: Fundamentals of Modern Manufacturing: Materials, Processes and Systems. Wiley, New Jersey (2010) 
2. Yusup, N., Mohd, A., Mohd, S.: Evolutionary Techniques in Optimizing Machining Parameters: Review and Recent Applications (2007-2011). Expert. Syst. Appl. 39, pp. 9909-9927 (2012)

3. Bharathi, S., Baskar, N.: Particle Swarm Optimization Technique for Determining Optimal Machining Parameters of Different Work Piece Materials in Turning Operation. Int. J. Adv. Manuf. Technol, 54, pp. 445-463 (2011)

4. Segura, C., Coello, C., Miranda, G., León, C.: Using Multi-Objective Evolutionary Algorithms for Single-objective Constrained and Unconstrained Optimization. Ann. Oper. Res., pp. 217-250 (2016)

5. Yildiz, A.: A New Hybrid Differential Evolution Algorithm for the Selection of Optimal Machining Parameters in Milling Operations. Appl. Soft. Comput., 13, pp. 1561-1566 (2013)

6. Mohd, A., Haron, H., Sharif, S.: Genetic Algorithm and Simulated Annealing to Estimate Optimal Process Parameters of the Abrasive Waterjet Machining. Eng. Comp., 27, pp. 251259 (2011)

7. Li, C., Xiao, Q., Tang, Y., Li, L.: A method Integrating Taguchi, RSM and MOPSO to CNC Machining Parameters Optimization for Energy Saving. J. Clean. Prod., 135, pp. $263-$ 275 (2016)

8. Kadirgama, K., Noor, M., Alla, A.: Response Ant Colony Optimization of End Milling Surface Roughness. Sensors, 10, pp. 2054-2063 (2010)

9. Samanta, S., Chakraborty, S.: Parametric Optimization of Some Non-Traditional Machining Processes using Artificial Bee Colony Algorithm. Eng. Appl. Art. Intell., 24, pp. 946-957 (2011)

10. Zavala, G., Nebro, A., Luna, F., Coello, C.: A survey of Multi-Objective Metaheuristics Applied to Structural Optimization. Struct. Multidisc. Optim., 49, pp. 537-558 (2014)

11. Yan, J., Li, L.: Multi-objective Optimization of Milling Parameters-the Trade-offs Between Energy, Production Rate and Cutting Quality. J. Clean. Prod., 52, pp. 462-471 (2013)

12. Wang, Q., Liu, F., Wang, X.: Multi-objective Optimization of Machining Parameters Considering Energy consumption. Int. J. Adv. Manuf. Technol., 71, pp. 1133-1142 (2014)

13. Kovacevic, M., Madic, M., Radovanovic, M., Rancic, D.: Software Prototype for Solving Multi-Objective Machining Optimization Problems: Application in Non-Conventional Machining Processes. Expert. Syst. Appl., 41, pp. 5657-5668 (2014)

14. Zhang, T., Owodunni, O., Gao, J.: Scenarios in Multi-objective Optimisation of Process Parameters for Sustainable Machining. Proceedia CIRP, 26, pp. 373-378 (2015)

15. Rezaee, A., Jasni, J.: Particle Swarm Optimization for Discrete Optimization Problems: A Review. Artif. Intell., 43, pp. 243-258 (2015) 